\title{
Possible extensions of the standard cosmological model: anisotropy, rotation, and magnetic field
}

\author{
M. Demiański \\ Institute of Theoretical Physics, University of Warsaw, 00-681 Warsaw, Poland \\ Department of Astronomy, Williams College, Williamstown, MA 01267, USA \\ A.G. Doroshkevich \\ Astro Space Center of Lebedev Physical Institute of Russian Academy of Sciences, \\ 117997 Moscow, Russia
}

\begin{abstract}
We show that the difference between the theoretically expected and measured by WMAP amplitude of the quadrupole fluctuations of $\mathrm{CMB}$ can be related to the impact of the anisotropic curvature of the homogeneous universe dominated by the dark energy. In such universe the matter expansion becomes practically isotropic just after the period of inflation and only at small redshifts the anisotropic expansion is generated again by the small curvature $\Omega_{K}=1-\Omega_{m}-\Omega_{\Lambda} \leq 10^{-4}$. For such models the possible deviations from the parameters derived for the standard cosmological model are evidently negligible but the correlations of large scale perturbations and distortions of their Gaussianity are possible. Such models are also compatible with existence of a homogeneous magnetic field and matter rotation which contribute to the low $\ell$ anisotropy and can be considered as "hidden parameters" of the model. Their influence can be observed as, for example, special correlations of small scale fluctuations and the Faraday rotation of the CMB and radiation of the farthest quasars. However, both the magnetic field and matter rotation require also modifications of the simple models of isotropic inflation and they change the evolutionary history of the early Universe.

PACS number(s): 98.80.Es.
\end{abstract}

\section{INTRODUCTION}

Recent rapid progress of observational cosmology resulted in formulation of the "Cosmological Standard Model" (CSM) (see, e.g., review by Springel, Frenk \& White 2006). This model successfully describes main observations at redshifts $0 \leq z \leq 10^{9}$ such as the primordial nucleosynthesis, the $\mathrm{CMB}$ fluctuations (WMAP, Hinshaw et al. 2006), the Large Scale Structure in the observed galaxy distribution (SDSS, Tegmark et al. 2004; 2dFGRS, Cole et al. 2005) and the redshift variations of brightness of SN Ia (Astier et al. 2006; Wood-Vasey et al. 2007).

The CSM provides us with a reasonable framework for its further extensions which will change and improve interpretation of some observational data but the well established characteristics of the basic model will remain unchanged. Some of such extensions were already considered during last years. Thus, Durrer, Kahniashvily \& Yates (1998), Kosovsky et al. (2005), Durrer (2007) and Kahniashvily \& Ratra (2007) have considered the CMB anisotropy generated by the magnetic field and, in particular, by Alfven waves. A possible observational manifestation of small scale entropy perturbations were discussed in Naselsky and Novikov (2002) and Doroshkevich et al. (2003). Both extensions can weakly distort the primordial nucleosynthesis and fluctuations of the $\mathrm{CMB}$ temperature and polarization but they do not change the basic parameters of the CSM.

On the contrary, attempts of Jaffe et al. (2005, 2006), Cayon et al. (2006), Bridges et al. (2006), McEwen et al. (2006) to exploit the Anisotropic Cosmological Models (ACM) with a large curvature to improve the interpretation of many anomalies of the observed maps of CMB fluctuations are evidently in contradiction with both the inflationary model and the observational upper limit for the spatial curvature of the Universe in CSM. More detailed analysis of this model (Naselsky \& Verkhodanov 2006) reveals also serious divergences between expected and observed phases of reconstructed harmonics what further decreases the appeal of such attempts to drastically change the interpretation of measured fluctuations.

The most active discussion is now concentrated around anomalies in the large scale fluctuations of the CMB temperature measured by WMAP. As is well known, for low $\ell$ multipoles there are serious deviations from Gaussianity which can be mostly caused by imperfections of the methods used for discrimination between the CMB and foreground (see, e.g., Naselsky et al. 2005 and references there). In particular, the "standard model" predicts the amplitude of quadrupole fluctuations at the 
level $\Delta T^{2}=1250 \mu K^{2}$ (Spergel et al. 2006) while the observed value provided by both COBE and WMAP measurements is only $\Delta T^{2}=249 \mu K^{2}$ (Hinshaw et al. 2006). Moreover, in several papers it was declared that the quadrupole and octopole moments are correlated with their two preferred planes surprisingly closely aligned. The preferred axis, dubbed the "axis of evil" by Land and Magueijo (2005), is found to be pointing towards Virgo and is close to the ecliptic pole (Oliveira-Costa et al. 2004). Detailed discussion of these problems can be found in Oliveira-Costa \& Tegmark (2006) where more than hundred references are summarized.

Here we cannot discuss these results and all the proposed so far interpretations of these peculiarities. Let us only note that the measured quadrupole fluctuations of the CMB are formed by a combined action of the suitable random inhomogeneities in the matter motion and distribution and of the possible regular factors such as the anisotropic curvature, homogeneous magnetic field and/or matter rotation. However, the extension of the CSM by incorporating these regular factors stimulates a renewed interest in the Anisotropic Cosmological Models.

The Anisotropic Cosmological Models (ACM) were quite popular in the $60^{t h}$ and $70^{t h}$ (see, e.g. Zel'dovich \& Novikov 1983) when scarcity of observational information have not restricted such discussions. As was established in many papers, for such models the strong anisotropic expansion at an early stage is incompatible with observational estimates of abundances of light elements. Early anisotropic expansion can be naturally suppressed at the period of inflation and after inflation the ACM becomes identical to the isotropic FriedmanRobertson-Walker (FRW) model with a negligible magnetic field and rotation. This means that the model proposed by Campanelli et al. (2006) is incompatible with the standard models of inflation (see also discussion below in Sec. 4).

However, the anisotropic curvature present in all ACM (with the exception of Bianchi type I models) decreases but does not disappear during the period of inflation. Almost all ACM with a suitably small anisotropic curvature are close to the isotropic FRW model at redshifts $z \gg 1$. In fact, the influence of the small anisotropic curvature on the cosmological recombination of hydrogen or the evolution of perturbations and galaxy formation is negligible. So, it does not change all conclusions of CSM related to the evolution of the Universe at $z \geq 1$ such as, in particular, the small scale CMB fluctuations. However, at redshifts $z \leq 1$ the anisotropic curvature generates again the regular weak anisotropy of the matter expansion which provides the regular contribution to the CMB quadrupole.

Here we illustrate this statement by considering the simplest ACM with the axial symmetry which provides the natural explanation of the deficit of power in the quadrupole mode. Even this model allows to introduce also the homogeneous magnetic field as a "hidden parameter" of the cosmological model. More complex ACMs allow also to introduce rotation of the Universe generalizing the well known cosmological model of Gödel (1952) (see, e.g., Ellis \& MacCallum 1969). Some published estimates related to such more complex ACM are shortly summarized in Sec. 4.

\section{ANISOTROPIC COSMOLOGICAL MODEL}

The simplest ACM is the Bianchi type III model which was discussed already in Schucking and Heckmann (1958), Kompaneets and Chernov (1965) and Doroshkevich (1965). Of course, this extension is not unique and more complex Bianchi models can be also considered leading to similar results (see, e.g., Doroshkevich et al. $1972 \mathrm{a}, \mathrm{b})$.

This ACM is described by the metric

$$
\begin{gathered}
d s^{2}=c^{2} d t^{2}-a^{2}(t)\left[d r^{2}+f^{2}(r) d \phi^{2}\right]-b^{2}(t) d z^{2}, \\
f(r)=\{\operatorname{sh} r ; r ; \sin r\},
\end{gathered}
$$

for models with negative, zero and positive curvatures. Here $c$ is the speed of light and the functions $a(t) \& b(t)$ describe the anisotropic expansion of the Universe.

For the standard matter the energy-momentum tensor is

$$
T_{i k}=(\varepsilon+p) u_{i} u_{k}-p g_{i k},
$$

where $\varepsilon \& p$ are the energy density and pressure of the matter. In this case the evolution is determined by three equations

$$
\begin{gathered}
\dot{\alpha}+\alpha(2 \alpha+\beta)+\delta a^{-2}=\Lambda+(\kappa / 2)(\varepsilon-p), \\
\dot{\beta}+\beta(2 \alpha+\beta)=\Lambda+(\kappa / 2)(\varepsilon-p), \\
\alpha^{2}+2 \alpha \beta+\delta a^{-2}=\Lambda+\kappa \varepsilon,
\end{gathered}
$$

where $\operatorname{dot}^{\circ}$ denotes the time derivative and

$$
\alpha=\dot{a} / a, \quad \beta=\dot{b} / b, \quad \kappa=8 \pi G / c^{4} .
$$

Here $\Lambda>0$ is the cosmological constant and $\delta=-1,0,1$ for the models with negative, zero and positive curvature (1).

For the most interesting cases, namely, the anisotropic inflation in the early Universe and matter dominated expansion $(p=0)$ describing the evolution of the Universe at redshifts $z \leq z_{e q} \approx 10^{4}$ simple solutions can be obtained. 


\section{Period of the anisotropic inflation}

Thus, near the singularity, for $t \rightarrow 0$, we can neglect the impact of the curvature and matter density and consider the influence of the cosmological constant only. In this case we get for $\Lambda=\Lambda_{\text {inf }}$ (Ellis \& MacCallum 1969; MacCallum 1971)

$$
\begin{gathered}
a(t)=a_{0} \operatorname{ch}^{2 / 3} \tau \cdot \operatorname{th}^{p_{1}} \tau, \quad b(t)=b_{0} \operatorname{ch}^{2 / 3} \tau \cdot \operatorname{th}^{p_{2}} \tau, \\
2 p_{1}+p_{2}=2 p_{1}^{2}+p_{2}^{2}=1, \quad \tau=t \sqrt{3 \Lambda_{\text {inf }}} / 2 .
\end{gathered}
$$

This expression generalizes the well known Kasner solution by including the impact of the cosmological constant $\Lambda_{\text {inf }}$. At $\tau \leq 1$ these expressions describe the usual anisotropic expansion which can be linked with the influence of the large scale gravitational waves (see, e.g., Belinsky et al. 1972, 1982; Doroshkevich e al. 1972 a, b; Zel'dovich \& Novikov 1983; Landau \& Lifschitz 1991). This anisotropic expansion is rapidly suppressed and at $\tau \geq 1$ we get almost isotropic expansion with

$$
a(t) \propto b(t) \propto \exp (2 \tau / 3), \quad a / b \rightarrow a_{0} / b_{0} .
$$

Non the less, in this model the anisotropy of the spatial curvature is retained. Moreover, the anisotropic expansion at $\tau \leq 1$ can generate anisotropy of the large scale perturbations what destroys their Gaussianity and produces correlations caused by the existence of preferred directions in the Universe.

Of course, these directions do not overlap with the dipole of the CMB, or with the direction to the Virgo or to the ecliptic pole and, so, these peculiarities cannot explain all the correlations among the measured low $\ell$ multipoles of the CMB. However, they demonstrate that the standard assumptions of isotropy and Gaussianity of all primordial perturbations could be too restrictive (see, e.g., Mukhanov 2005, Sec. 5).

\section{Period of the matter dominated expansion}

For the later period of the cosmological expansion when we can neglect the impact of the pressure $(p \ll \varepsilon)$ the solutions of the system (4) can be written as follows:

$$
3 \alpha^{2}=H_{0}^{2}\left[\Omega_{m}(1+z)^{3}+\Omega_{\Lambda}+\Omega_{K}(1+z)^{2}\right],
$$

where $H_{0} \& z=a(0) / a(t)-1$ are the Hubble constant and the redshift measured in the plane of symmetry, $\Omega_{m} \& \Omega_{\Lambda}$ are dimensionless density of the Dark Matter and Dark Energy and

$$
\Omega_{K}=1-\Omega_{m}-\Omega_{\Lambda}, \quad \Omega_{K} \propto-\delta,
$$

characterizes the curvature of the Universe. Integration of (6) links the scale factor $a$ with time $t$. Evidently, at the matter dominated stage, $(1+z)^{3} \geq \Omega_{\Lambda} / \Omega_{m}$, we have $a \propto t^{2 / 3}$ and we recover the isotropic solution.

The anisotropy of expansion is measured by the ratio $a / b$ :

$$
(\alpha-\beta)^{\cdot}+(\alpha-\beta)(2 \alpha+\beta)=-\delta / a^{2} .
$$

For small $\left|\Omega_{K}\right| \ll 1$ and $|a / b-1| \ll 1$ we get

$$
\ln \left(\frac{a}{b}\right) \approx \Omega_{K} \int_{1+z}^{\infty} \frac{\xi^{2} d \xi}{\sqrt{\Omega_{m} \xi^{3}+\Omega_{\Lambda}}} \int_{\xi}^{\infty} \frac{\eta^{-2} d \eta}{\sqrt{\Omega_{m} \eta^{3}+\Omega_{\Lambda}}}
$$

For $(1+z)^{3} \gg\left(\Omega_{\Lambda} / \Omega_{m}\right) \geq 0$ we have

$$
\ln (a / b)=0.4\left(\Omega_{K} / \Omega_{m}\right)(1+z)^{-1}
$$

what corresponds to the growing mode of the density perturbations. For the accepted in CSM values of

$$
\Omega_{\Lambda} \approx 0.7, \quad \Omega_{m} \approx 0.3, \quad\left(\Omega_{m} / \Omega_{\Lambda}\right)^{1 / 3} \approx 0.754,
$$

we get from (8) for $z=0$ and $T \approx 2.7 K$

$$
\begin{gathered}
\Theta_{K}=\ln (a / b) \approx a / b-1 \approx 0.77 \Omega_{K}, \quad\left|\Omega_{K}\right| \ll 1, \\
\Delta T / T=\left\{-\Theta_{K} / 3,-\Theta_{K} / 3,2 \Theta_{K} / 3\right\},
\end{gathered}
$$

for the relative temperature fluctuations along the coordinate axes (1). As it follows from (7, 10) we have $\Theta_{K}=0$ for the flat model with $f(r)=r, \delta=\Omega_{K}=0$.

This result shows that the small positive or negative curvature provides us with the reasonable quadrupole anisotropy of the CMB temperature. In galactic coordinates the orientation of this quadrupole is arbitrary while its amplitude depends upon $\Omega_{K}$. The required curvature is quite small because the expected $\left|\Theta_{K}\right| \sim$ $\left|\Omega_{K}\right| \sim \sqrt{\left\langle(\Delta T / T)^{2}\right\rangle} \sim 10^{-5}$. For negative curvature $\Omega_{K}>0$, the expansion along $z$-axis is slower than that in the plane of symmetry and $\Theta_{K}>0$. For small positive curvature $\Omega_{K}<0$, the expansion along $z$-axis is faster than that in the plane of symmetry and $\Theta_{K}<0$.

At the same time it is evident that the required curvature $\left|\Omega_{K}\right|<10^{-5}$ is within range of $\sim 10 \%$ precision achieved by available measurements of cosmological parameters (see, e.g., Hinshaw et al. 2006).

\section{QUADRUPOLE ANISOTROPY OF CMB}

As is well known, the five quadrupole coefficients are linked with the components of a symmetric traceless tensor $Q_{i j}$

$$
\begin{aligned}
& \frac{Q_{11}}{\sqrt{2}}=a_{2,2}-\frac{a_{2,0}}{\sqrt{6}}, \frac{Q_{12}}{\sqrt{2}}=-a_{2,-2}, \quad \frac{Q_{13}}{\sqrt{2}}=-a_{2,-1},(11 \\
& \frac{Q_{22}}{\sqrt{2}}=-a_{2,2}-\frac{a_{2,0}}{\sqrt{6}}, \quad \frac{Q_{23}}{\sqrt{2}}=-a_{2,1}, \quad \frac{Q_{33}}{\sqrt{2}}=2 \frac{a_{2,0}}{\sqrt{6}} .
\end{aligned}
$$


This representation allows us to find the principle values of this tensor, $\lambda_{i}$, and their orientation in the galactic coordinates $l \& b$. With the standard definitions we have for the 3 years ILC measurements (Hinshaw et al. 2006):

$$
\begin{aligned}
& \lambda_{1}=27.1 \mu K, \quad(l, b)=\left(-0.8^{\circ} \pm 13^{\circ}, 63.3^{\circ} \pm 1^{\circ}\right), \\
& \lambda_{2}=12.9 \mu K, \quad(l, b)=\left(15.5^{\circ} \pm 3^{\circ}, 25.8^{\circ} \pm 1.2^{\circ}\right),(12) \\
& \lambda_{3}=-40 \mu K, \quad(l, b)=\left(-77.6^{\circ} \pm 5^{\circ}, 6.5^{\circ} \pm 4^{\circ}\right),
\end{aligned}
$$

with

$$
I_{2} / 5=-\left(\lambda_{1} \lambda_{2}+\lambda_{1} \lambda_{3}+\lambda_{2} \lambda_{3}\right) / 5=\Delta T^{2}=250 \mu K^{2} .
$$

These orientations differ from both the dipole direction

$$
(l, b)_{D}=\left(-96^{\circ}, 48^{\circ}\right),
$$

and the possible but quite arbitrary orientation of the quadrupole introduced by Oliveira-Costa et al. (2004)

$$
(l, b)=\left(-110^{\circ}, 60^{\circ}\right) .
$$

The actual orientations of the observed octopole and higher multipoles can be reconstructed also using the principle axes of corresponding symmetric tensors.

The observed quadrupole is a superposition of the regular and the random ones. The regular quadrupole is related to the anisotropic expansion of the Universe while the random one is generated by the initial perturbations. Relative orientation of these quadrupoles is arbitrary and, so, we can obtain only the rough estimate of the required regular quadrupole and the curvature of the Universe. Comparing the observed and expected quadrupoles we get

$$
\left|\Theta_{K}\right| \approx\left|\Omega_{K}\right| \sim 10^{-5}-10^{-4}
$$

For example, for the expected value $\Delta T^{2}=1250 \mu \mathrm{K}$, $\lambda_{i}$ as given by (12) and for the two special orientations of the regular quadrupole (11) along the principle axes (12) we get instead of (10) for the relative quadrupole fluctuations:

$$
\begin{gathered}
\frac{\Delta T}{T}=\left\{\frac{\lambda_{1}}{\sqrt{5} T}+\frac{2 \Theta_{1}}{3}, \frac{\lambda_{2}}{\sqrt{5} T}-\frac{\Theta_{1}}{3}, \frac{\lambda_{3}}{\sqrt{5} T}-\frac{\Theta_{1}}{3}\right\}, \\
\Theta_{1}=\Theta_{K} \approx\{14.6 ;-28.1\} \cdot 10^{-6}, \\
\frac{\Delta T}{T}=\left\{\frac{\lambda_{1}}{\sqrt{5} T}-\frac{\Theta_{3}}{3}, \frac{\lambda_{2}}{\sqrt{5} T}-\frac{\Theta_{3}}{3}, \frac{\lambda_{3}}{\sqrt{5} T}+\frac{2 \Theta_{3}}{3}\right\}, \\
\Theta_{3}=\Theta_{K} \approx\{32.5 ;-12.7\} \cdot 10^{-6} .
\end{gathered}
$$

This choice of the quadrupole orientation is consistent with the possible correlations of the low $\ell$ perturbations and the spatial curvature discussed in Sec. 2.1. These results are consistent with the available estimates of the cosmological parameters (see, e.g. Spergel et al. 2006) and weakly depend upon the accepted values of $\Omega_{\Lambda}$ and $\Omega_{m}$.

\section{MODELS WITH THE MAGNETIC FIELD AND ROTATION}

The small anisotropic curvature can only generate the large scale anisotropy of the CMB. However, combined with the matter rotation and/or the magnetic field such anisotropy will also successfully distort the Gaussianity of small scale CMB fluctuations and, in particular, generate correlations between different modes of these fluctuations. Already available restrictions of the admissible amplitude of the rotation and magnetic field show that, as a rule, their interactions with the cosmological plasma leads to several observable effects. However, potentialities of such interactions are not exhausted and, in spite of their incompatibility with the standard inflation models, they deserve further discussion.

\section{Influence of the magnetic field}

As was shown in Zel'dovich (1965), Doroshkevich (1965), and Thorne (1967) the model (1) can include a homogeneous magnetic field along the $z$ axis. Presently accepted estimates of this field $B(z=0) \leq 10^{-9} G$ are obtained from the observed regular magnetic fields within galaxies $(\sim 10 \mu G)$ and clusters of galaxy $(\sim 0.1 \mu G)$ (see reviews by Widrow 2002; and Semikoz \& Sokoloff 2005). However, such fields do not noticeably influence the expansion rate of the Universe and the CMB fluctuations because their energy density, $w(z)=B^{2}(z) / 8 \pi \propto$ $(1+z)^{4}$, is small in comparison with the energy density of $\mathrm{CMB}, \varepsilon_{\text {rad }} \approx 4 \cdot 10^{-13}(1+z)^{4} \mathrm{erg} / \mathrm{cm}^{3}$,

$\Omega_{B}=\kappa c^{2} \frac{w(0)}{3 H_{0}^{2}} \leq 10^{-11}\left(\frac{B(0)}{10^{-9} G}\right)^{2}, \frac{w}{\varepsilon_{\text {rad }}} \approx 10^{5} \Omega_{B}(17)$

Indeed, the contribution of the weak magnetic field is described by the term $\Omega_{B}(1+z)^{4}$ in Eq. (6) and instead of (8) we will have for the function $\Theta=\Theta_{B}$

$\Theta_{B}=2 \Omega_{B} \int_{1+z}^{1+z_{r}} \frac{\xi^{2} d \xi}{\sqrt{\Omega_{m} \xi^{3}+\Omega_{\Lambda}}} \int_{\xi}^{1+z_{r}} \frac{d \eta}{\sqrt{\Omega_{m} \eta^{3}+\Omega_{\Lambda}}}(18)$

where $z_{r} \approx 10^{3}$ is redshift of the hydrogen recombination and for $z \geq z_{r}$ the radiation can be considered as isotropic. As is seen from (18), the amplitude of regular quadrupole is

$$
\Theta_{B} \approx \frac{4 \Omega_{B}}{3 \Omega_{m}} z_{r} \approx 4 \Omega_{B} \cdot 10^{3} \leq 4 \cdot 10^{-8}\left(\frac{B(0)}{10^{-9} G}\right)^{2},
$$

and at least a field $B \geq 10^{-7} G$ is required to reproduce the expected regular quadrupole (1516). More details can be found in Durrer (2007) and Kahniashvili \& Ratra (2007). 


\section{Influence of the matter rotation}

Many homogeneous models are compatible also with the matter rotation (see, e.g., Ellis \& MacCallum 1969; Barrow et al. 1985). The evolution of slow rotation is quite similar to that of the magnetic field and is determined by the angular momentum conservation law (see, e.g., Landau \& Lifschitz 1991). Thus, for the matter dominated Universe at $z \leq z_{e q} \approx 10^{4}$ the angular velocity of matter, $\omega(z)$, is changing as

$$
\frac{\omega(z)}{H(z)} \approx \sqrt{\frac{1+z}{\Omega_{m}}} \frac{\omega(0)}{H_{0}},
$$

where $\omega^{2}(z)=\omega_{\nu}(z) \omega^{\nu}(z)$ and $\omega_{\nu}$ and $\omega^{\nu}$ are the covariant and contravariant components of the angular velocity. This relation shows that at the matter dominated period the slow rotation does not influence the evolution of the Universe. At the radiation dominated period for $z \geq z_{e q} \approx 10^{4}$ we have

$$
\omega(z) / H(z) \propto(1+z)^{-1},
$$

and, so, the maximal value of $\omega / H$ is achieved at $z \sim$ $z_{e q} \approx 10^{4}$. However, the rotation influences the evolution of the models near the singularity (see, e.g., Ellis \& MacCallum 1969).

The observed rotation of galaxies corresponds to

$$
\omega(0) / H_{0} \leq 10^{-2},
$$

but the measured $\mathrm{CMB}$ anisotropy leads to stronger restrictions. Thus, for one of the Bianchi VI models discussed in Ellis \& MacCallum (1969) we get for the quadrupole temperature fluctuations

$$
\Theta_{\omega}=\Theta_{K}-\frac{2 z_{r}^{3}}{9 \Omega_{m}}\left(\frac{\omega(0)}{H_{0}}\right)^{2},
$$

where $\Theta_{K}>0$ is given by (10) and $z_{r} \approx 10^{3}$ is the redshift of recombination epoch. It is interesting that in this case the influence of the curvature and rotation can partly compensate each other and, therefore, $\Omega_{K}>10^{-5}$ and $\omega(0) / H_{0}>10^{-7}$ can be considered.

More complex pattern appears for the Bianchi VII model where together with quadrupole fluctuations also higher multipoles correlated with the quadrupole are generated. Detailed analysis of these fluctuations (Barrow et al. 1985; Jaffe et al. 2005, 2006; Ghosh et al. 2006) leads to the following estimates

$$
\omega(0) / H_{0} \leq 4 \cdot 10^{-5}-10^{-6},
$$

and even in this model we can expect that $\omega(z) / H(z) \ll$ 1 at all redshifts.

These results indicate that rotation and/or magnetic field can influence the evolution of the Universe mainly during the radiation dominated period at redshifts $z \geq$ $z_{e q} \approx 10^{4}$ or at the period of cosmological recombination $z \sim z_{r} \approx 10^{3}$.

\section{Observational manifestations}

The most interesting observational manifestations of both factors are the possible rotation of the CMB polarization, distortions of its Gaussianity and the power spectra of the temperature and polarization at small and large scales, and, in particular, intermixture of the "E" and "B" modes of the CMB polarization (see, e.g., Kosowsky \& Loeb 1996; Harari et al. 1997; Campanelli et al. 2004; Kosowsky et al. 2005; Subramanian 2006; Giovannini 2006). In fact, the expected Faraday rotation of the $\mathrm{CMB}$ is estimated to be

$$
\left\langle\varphi^{2}\right\rangle^{1 / 2} \approx 1 .^{\circ} 6\left(\frac{B}{10^{-9} G}\right)\left(\frac{\lambda}{1 \mathrm{~cm}}\right)^{2} .
$$

Another observational manifestation of the magnetic field is the Faraday rotation of the radiation of farthest quasars (see, e.g., Widrow 2002; Hutsemekers et al. 2005; Semikoz \& Sokoloff 2005).

Durrer et al. (1998) have considered the distortions of small scale CMB fluctuations caused by the interaction of CMB with the possible Alfven waves and even find similar correlations in the observed temperature maps of WMAP (Durrer 2007; Kahniashvili \& Ratra 2007). However, such identifications are in question (Naselsky et al. 2004).

It is well known that both the rotation and magnetic fields are strongly suppressed in the models of standard inflation (see, e.g., Widrow 2002). This means that serious discussions of ACM with rotation and magnetic field require suitable modifications of the inflationary models, or alternatively generation of the rotation and/or magnetic field in the course of evolution during or after inflation (see, e.g., Turner and Widrow 1988; Ratra 1992; Lasenby \& Doran 2004).

\section{DISCUSSION}

The "Cosmological Standard Model" with inflation at the earlier period of cosmological expansion and domination of the cosmological constant at small redshifts significantly extends the class of cosmological models compatible with presently available observational restrictions. In particular, the recent discussion of the high precision WMAP measurements (see, e.g., Oliveira-Costa \& Tegmark 2006 and references there) points out that, perhaps, some of the observed peculiarities can be successfully interpreted in the framework of anisotropic cosmological models rather than in the Friedman-RobertsonWalker cosmology. It is also important that such homogeneous anisotropic models allow to include into consideration the primordial rotation and magnetic fields.

The anisotropic cosmological models were popular in the $60^{t h}$ and earlier $70^{\text {th }}$ when attention was mostly 
focused on models that incorporated the FriedmanRobertson-Walker universe with the isotropic expansion (Zel'dovich \& Novikov 1983). Now we see (5) that the simple models of inflation successfully suppress the initial anisotropy linked with an expansion, magnetic field or rotation and provide almost isotropic expansion of the Universe down to small redshifts.

On the other hand, at small redshifts the cosmological constant efficiently suppresses the impact of the moderate anisotropic curvature and makes the expansion almost isotropic for a wide class of Bianchi models. Our estimates (8, 10, 14-15) demonstrate that the required deviations from the flat isotropic Universe could be quite small and their observational manifestations invite further careful study. Non the less, the consequences of such extensions are quite important because now the wide set of Bianchi models can be again used to explain various observed manifestations of the large scale anisotropy. In particular, in such models we can expect that

1. Distortions of Gaussianity of large scale perturbations and the anisotropy of their power spectrum lead to corresponding distortions of low $\ell$ random multipoles and their possible correlations.

2. For models with small spatial curvature the regular quadrupole term dominates.

3. For the $\mathrm{VII}_{0}$ Bianchi model with rotating matter the regular octopole and higher multipoles correlated with quadrupole are generated.

4. The magnetic field generates noticeable non Gaussianity in the small scale CMB fluctuations and leads to intermixture of the "E" and "B" modes of CMB polarization.

In the flat Bianchi type I model the anisotropy is related to the initial anisotropic expansion only and it rapidly increases with redshift. So, for this model only negligible anisotropy is compatible with the standard inflationary models and the cosmological nucleosynthesis. Because of this restriction and as was found above (Sec. 4.1) the simple elliptical model discussed in Campanelli et al. (2006) cannot provide the adequate explanation of the anisotropy of the CMB. At the same time, discussion in Naselsky \& Verkhodanov (2006) demonstrates the limited potentialities of the anisotropic cosmological models with the large spatial curvature (Jaffe et al. 2005, 2006; Cayon et al. 2006; Bridges et al. 2006; McEwen et al. 2006) for description of the WMAP observations of low $\ell$ multipoles.

Up to the present time analysis of the CMB fluctuations provides us with the best limitations of possible distortions of the standard cosmological model. In spite of such limitations the discussed extension of the cosmological models allows us to include into consideration as "hidden" parameters not only the anisotropic curvature and the entropy perturbations but, perhaps, also the magnetic field and rotation of the Universe, what implies various observational manifestations discussed above (Sec. 4). These effects could be revealed by high precision measurements of the Planck mission and detailed high precision observations of distant quasars. However, we still do not have reasonable models of inflation that allow generation of magnetic fields (see review in Widrow 2002; Semikoz \& Sokoloff 2005) and the rotation during or just after inflation.

\section{ACKNOWLEDGMENTS}

This paper was supported in part by the Polish State Committee for Scientific Research grant Nr.1-P03D-01426 and Russian Found of Fundamental Investigations grant Nr.05-02-16302. AD thanks Yu. Gnedin, B. Komberg, V. Lukash and P. Naselsky for useful comments. We should like to thank the anonymous referee for useful comments.

[ 1] Astier P. et al., 2006, A\&A., 447, 31

[2] Barrow J.D., Juszkiewicz R., Sonoda D.H., 1985, MNRAS, 213, 917

[ 3] Belinsky V., Lifschitz E., Khalatnikov I., 1972, Sov. Phys. JETP, 35, 83831

[ 4] Belinsky V., Lifschitz E., Khalatnikov I., 1982, Adv. in Phys., 31, 639

[ 5] Bridges M. et al., 2006, MNRAS, submit., astro-ph/0605325

[6] Campanelli L., Dolgov A., Giannotti M., Villante F.L., 2004, ApJ, 616, 1

[ 7] Campanelli L., Cea P., Tedesco L., 2006, Phys. Rev. Lett. 97, 131302

[ 8] Cayon L., Banday A., Jaffe T., Eriksen H., Hansen F., Gorski K., Jin J., 2006, MNRAS, 369, 598

[ 9] Doroshkevich A., 1965, Astrophysics, 1, 138

[10] Doroshkevich A., Lukash V., Novikov I., 1974a, Sov. Phys. JETP, 37, 739

[11] Doroshkevich A., Lukash V., Novikov I., 1974b, Sov.Astron., 18, 554

[12] Doroshkevich A., Naselsky I., Naselsky P., Novikov I., 2003, ApJ, 586, 709

[13] Durrer R., Kahniashvili T., Yates A., 1998, Phys. Rev., D58, 123004

[14] Durrer R., 2007, New Astron. Rev., 51, 275

[15] Ellis G.F.R. \& MacCallum M.A.H., 1969, Comm. Math. Phys., 12, 108

[16] Ghosh T., Hajian A., Souradeep T., astro-ph/0604279

[17] Giovannini M., 2006, Class. Quant. Grav., 23, 4991

[18] Gödel K., 1952, Proc. Intern. Congr. Math., Cambridge, Mass., 1, 175

[19] Harari D., Hayward J., Zaldarriaga M., 1997, Phys. Rev., D55, 1841

[20] Heckmann O., Schucking E., 1958, Solvay Conference Report, Bruxells, p. 149, Edition Stops; 1962, Chap 11 
of Gravitation: An introduction to current research, ed. Witten, New York,: Willey

[21] Hinshaw G. et al., 2006, astro-ph/0603451

[22] Hutsemekers D., Cabanac R., Lamy H., Sluse D., 2005, astro-ph/0507274

[23] Jaffe T., Banday A., Eriksen H., Gorski K., Hansen F., 2005, ApJ, 643, 616

[24] Jaffe T., Banday A., Eriksen H., Gorski K., Hansen F., 2005, ApJ, 644, 701

[25] Jaffe T., Banday A., Eriksen H., Gorski K., Hansen F., 2006 astro-ph/0606046

[26] Kahniashvili T., Ratra B., 2007, Phys. Rev., D75, 023002

[27] Kompaneets A., Chernov A., 1965, Sov. Phys. JETP, 20, 1303

[28] Kosowsky A. \& Loeb A., 1996, ApJ, 469, 1

[29] Kosowsky A., Kahniashvili T., Lavrelashvili G., Ratra B., 2005, Phys. Rev., D71, 043006

[30] Land K., Magueijo J., 2005, Phys. Rev. Lett., 95, 071301

[31] Landau L. \& Lifschitz E., 1991, The classic field theory, Werlag Programm Physik

[32] Lasenby A.\& Doran C., 2004, astro-ph/0411579

[33] MacCallum M.A.H., 1971, Comm. Math. Phys., 20, 57

[34] McEwen J.D., Hobson M.P., Lasenby A.N., Mortlock D.J., 2005, MNRAS, 369, 1858

[35] Mukhanov V., 2005, Physical foundations of cosmology, Cambridge University Press, Cambridge.

[36] Naselsky P., Novikov I., 2002, MNRAS, 334, 137
[37] Naselsky P., Chiang L-Y., Olsen P., Verkhodanov O., 2004, ApJ, 615, 45

[38] Naselsky P., Chiang L-Y., Olsen P., Novikov I., 2005, Phys. Rev., D72, 063512

[39] Naselsky P., Verkhodanov O., 2006, MNRAS, submit., astro-ph/0609409

[40] de Oliveira-Costa A., Tegmark M., Zaldarriaga M., Hamilton A., 2004, Phys. Rev., D69, 063516

[41] de Oliveira-Costa A., Tegmark M., 2006, Phys. Rev., D74, 023005

[42] Ratra B., 1992, ApJ, 391, L1

[43] Semikoz V., Sokoloff D., 2005, IGMP, 14, 1839

[44] Spergel D. N., et al., 2006, astro-ph/0603449

[45] Springel V., Frenk C., White S.D.M., 2006, Nature, 440, 1137; astro-ph/0604561

[46] Subramanian K., 2006, Astron.Nachr. in press, astro-ph/0601570

[47] Thorne K.S., 1967, ApJ, 148, 51

[48] Turner M.S., Widrow L.M., 1988, Phys. Rev., D37, 2743

[49] Widrow L.M., 2002, Rev. Mod. Phys, 74, 775

[50] Woody-Vasey W. M. et al., 2007, astro-ph/0701041

[51] Zel'dovich Ya.B., 1965, Sov. Phys. JETP, 48, 986

[52] Zel'dovich Ya.B., Novikov I.D., 1983, The Structure and Evolution of the Universe, University of Chicago Press, Chicago. 